\title{
Análise das interações medicamentosas e perfil epidemiológico de indivíduos com diabetes mellitus na atenção primária
}

Analysis of drug interactions and epidemiological profile of individuals with diabetes mellitus in primary care

Análisis de interacciones farmacológicas y perfil epidemiológico de individuos con diabetes mellitus en atención primaria

\section{Leila Zanatta ${ }^{\mathrm{I}}$, Fernanda Norbak Dalla Cort ${ }^{\mathrm{II}}$, Nathália Silva Mathias ${ }^{\mathrm{III}}$, Carla Argenta ${ }^{\mathrm{IV}}$}

Resumo: Objetivo: analisar as interações medicamentosas e o perfil epidemiológico de indivíduos com diabetes mellitus (DM). Método: estudo quantitativo com aplicação de questionário a 42 pacientes com DM pertencentes a um Centro de Saúde da Família (CSF). Os dados foram coletados em 2018, em três etapas: encontro no CSF, visitas domiciliares e busca em prontuário eletrônico; seguido de análise das interações medicamentosas nas bases Drug Interactions Checker Drug Information e DrugBank. Resultados: a idade média dos pacientes foi de 68,36 anos. O número total de associações entre fármacos foi de 1355 (média de 32,26/paciente). O total de medicações que interagem foi de 479 (11,40 interações/paciente). Em 65\% as combinações não interagiram, 4\% foram interações leves, 26,05\% moderadas e 1,70\% graves. Conclusão: a quantidade de interações medicamentosas é expressiva, predominando as de grau moderado. A idade dos pacientes e presença de comorbidades podem estar associadas à polimedicação, contribuindo para ocorrência dessas interações.

Descritores: Diabetes mellitus; Hipoglicemiantes; Enfermagem; Polimedicação

Abstract: Aim: to analyze drug interactions and the epidemiological profile of individuals with diabetes mellitus (DM). Method: quantitative study with the application of a questionnaire to 42 patients with DM belonging to a Family Health Center (FHC). Data were collected in 2018, in three stages: meeting at the CSF, home visits and

\footnotetext{
I Farmacêutica, Doutora em Farmácia, Universidade do Estado de Santa Catarina - UDESC, Chapecó, SC, Brasil. E-mail: leila.zanatta@gmail.com, ORCID: https://orcid.org/0000-0003-0935-4190

II Enfermeira, Universidade do Estado de Santa Catarina - UDESC, Chapecó, SC, Brasil. E-mail: fernandanorbak@outlook.com, ORCID: https://orcid.org/0000-0003-1609-4972

III Enfermeira, Universidade do Estado de Santa Catarina - UDESC, Chapecó, SC, Brasil. E-mail: nathalya.mathiass@hotmail.com ORCID: https://orcid.org/0000-0003-3352-5885

IV Enfermeira, Doutora em Enfermagem, Universidade do Estado de Santa Catarina - UDESC, Chapecó, SC, Brasil. E-mail: carla.argenta@udesc.br ORCID: https://orcid.org/0000-0002-9729-410X
} 
Análise das interações medicamentosas e perfil epidemiológico de indivíduos com... $\mid 2$

searching electronic medical records; followed by analysis of drug interactions in the Drug Interactions Checker Drug Information and DrugBank databases. Results: the mean age of the patients was 68.36 years. The total number of associations between drugs was 1355 (mean 32.26 / patient). The total number of medications that interact was 479 (11.40 interactions /patient). In 65\% the combinations did not interact, $4 \%$ were mild interactions, $26.05 \%$ moderate and $1.70 \%$ severe. Conclusion: the number of drug interactions is significant, with a moderate degree predominating. The age of patients and the presence of comorbidities can be associated with polymedication, contributing to the occurrence of these interactions.

Descripors: Diabetes Mellitus; Hypoglycemic Agents; Nursing; Polypharmacy

Resumén: Objetivo: analizar como interacciones medicamentosas y el perfil epidemiológico de individuos con diabetes mellitus (DM). Método: estudio cuantitativo con aplicación de cuestionario a 42 pacientes con DM pertencentes en el Centro de Salud de la Familia (CSF). Os dados foram coletados em 2018, em três etapas: encontro no CSF, visitas domiciliares e busca em prontuário eletrônico; seguido de análise das interações medicamentosas nas bases Comprobador de interacciones de medicamentos Información sobre medicamentos e DrugBank. Resultados: una idade media dos pacientes fe de 68,36 años. Número total de asociaciones entre religiones de 1355 (medios de 32.26/paciente). O total de medicamentos que interagencian la fe de 479 (11.40 interacciones/paciente). Em $65 \%$ como combinações não interagiram, $4 \%$ foram interações leves, $26.05 \%$ moderadas y $1.70 \%$ grave. Conclusión: una cantidad de interacõs medicamentosas é expressiva, predominando como de grau moderado. A idade dos pacientes y presença de comorbidades podem estar asociado a polimedicação, contribindo para ocorrência dessas interações.

Descriptores: Diabetes Mellitus; Hipoglucemiantes; Enfermería; Polifarmacia

\section{Introdução}

O Diabetes Mellitus (DM) é caracterizado por um conjunto de sinais e sintomas decorrentes da deficiência da regulação do metabolismo de carboidratos, lipídeos e proteínas, causado pela inexistência da secreção de insulina ou ainda, pela diminuição da sensibilidade dos tecidos à mesma. ${ }^{1}$ Dentre os diversos tipos de DM os mais comuns são o DM tipo 1 e DM tipo 2. O tipo 1 ocorre pela ausência de secreção de insulina, sendo subdividido em A1 e A2 procedente da presença ou ausência de autoanticorpos circulantes. ${ }^{1} \mathrm{O}$ DM tipo 2 , inicialmente é provocado pela diminuição da sensibilidade dos tecidos ao efeito metabólico da insulina, a qual é chamada de resistência insulínica. ${ }^{1}$ Seu tratamento pode ser de caráter medicamentoso ou não medicamentoso, que envolve a prática de atividade física, hábitos alimentares saudáveis, interrupção do tabagismo e o controle da glicemia. 
Devido à cronicidade deste agravo, esses indivíduos possuem tendência a deterioração clínica com o passar do tempo, propiciando complicações derivadas de fatores de risco como a idade avançada somada ao DM, ou ainda, a falta do controle dos níveis glicêmicos. ${ }^{2}$ Diante disso, esses fatores determinam a necessidade de um olhar ampliado para o surgimento de possíveis interações medicamentosas, já que as mesmas ocorrem quando se tem a associação de medicamentos, cujos efeitos e reações podem ser alteradas em decorrência dessas combinações. ${ }^{3}$ O uso combinado de dois ou mais fármacos pode ser utilizado na terapêutica para a potencialização do efeito farmacológico e assim maximizar a eficácia do tratamento, entretanto, pode causar severa toxicidade no organismo, bem como, a ineficácia dos fármacos. ${ }^{4}$

Neste contexto, se destaca a polifarmácia, que se caracteriza pelo uso de cinco ou mais medicamentos devido a um ou múltiplos agravos à saúde. ${ }^{4} \mathrm{~A}$ integração de vários fármacos, viabiliza o aumento dos erros de prescrição médica, intensifica as interações medicamentosas, bem como os riscos de efeitos adversos, consumo inadequado, aumenta probabilidade de falta de adesão ao tratamento e maximiza as chances de morbimortalidade. ${ }^{4}$

Sendo assim, a enfermagem, juntamente com os demais profissionais da saúde, acompanha e orienta os pacientes com DM em diversas dimensões tais como, em ações educativas, na prevenção de agravos associados ao DM, buscando o alinhamento entre o tratamento e realidade situacional do indivíduo e sua família, expandido o olhar de forma integral ao usuário, incentivando a importância do autocuidado, trazendo orientações sobre tratamento, seja ele medicamentoso ou não, e estimulando o processo de autonomia do mesmo. ${ }^{5}$

Com base nas informações descritas surgiu a seguinte pergunta de pesquisa: qual a frequência e gravidade das interações medicamentosas em pacientes com DM atendidos em um Centro de Saúde da Família (CSF)? Na busca de respostas para essa pergunta o presente trabalho visa analisar as interações medicamentosas e o perfil epidemiológico de indivíduos com diabetes mellitus. 
Análise das interações medicamentosas e perfil epidemiológico de indivíduos com... | 4

\section{Método}

Trata-se de um estudo transversal, de abordagem quantitativa, realizado no segundo semestre de 2018, envolvendo 42 indivíduos com DM tipo 1 e 2 cadastrados em um CSF no município de Chapecó, Santa Catarina. O tamanho da amostra foi determinado com base no estudo que ressalta que a amostra deve ter um número mínimo cinco vezes maior do que o número de variáveis a serem analisadas. ${ }^{6}$

Aos usuários com DM que abrangiam a área do CSF selecionado, foi entregue por meio das Agentes Comunitárias de Saúde (ACS), um convite impresso para a participação do encontro a ser desenvolvido no Centro no qual realizou-se a coleta de dados. Não atingindo o número de participantes desejável, houve a necessidade de realização da coleta por meio de visitas domiciliares. As visitas aos usuários foram realizadas segundo o cronograma das ACS, estabelecido pela enfermeira coordenadora do serviço, que destinou aleatoriamente os pacientes com diagnóstico de DM tipo 1 e 2 cadastrados naquele território e que atendessem aos critérios de inclusão para participação na pesquisa. As visitas foram realizadas até que se atingisse o número amostral calculado. Além disso, para complementar os dados do questionário que os pacientes não conseguiram responder, realizou-se a busca em prontuário eletrônico a partir do seu número de cadastro e/ou nome completo.

Como critérios de inclusão, foram aceitos pacientes de ambos os sexos, que residissem no município em questão e que frequentassem o CSF, abrangendo participantes maiores de 18 anos, não estipulando uma idade máxima. Outrossim, ser diagnosticado com DM e estar usando dois ou mais medicamentos. Já o critério de exclusão, englobou pacientes que possuíssem algum déficit cognitivo, que os impedisse de responder às questões individualmente ou mesmo com o auxílio dos pesquisadores. 
Os dados referentes ao perfil sociodemográfico, de hábitos de vida, condições de saúde e de interações medicamentosas foram obtidos por meio da aplicação de um questionário que foi construído e adaptado pelos pesquisadores com base na literatura..$^{7-8} \mathrm{O}$ mesmo continha questões mistas (abertas e fechadas) e foi aplicado aos pacientes com DM presentes no encontro. Num primeiro momento foram abordados assuntos de relevância ao DM e posteriormente os participantes foram convidados a participar da referida pesquisa.

Dentre as características avaliadas por meio do questionário estão: 1) características sociodemográficas (sexo, idade, estado civil, raça, escolaridade, profissão); 2) condições de saúde; 3) hábitos de vida (uso de chás, prática de atividade física, alimentação, tabagismo, ingestão de bebida alcoólica); 4) cuidados relacionados à doença (monitorização da glicemia, necessidade de realização de procedimentos específicos por conta do diabetes, tempo de diagnóstico, frequência das consultas, dificuldade de cicatrização); 5-8) tratamento medicamentoso (número e tipo de medicamentos de uso habitual ou contínuo, dosagem, prática de automedicação).

Todos os dados coletados foram compilados em planilhas Microsoft $^{\oplus}$ Excel e apresentados como frequência absoluta e relativa ou média. Para a análise das interações medicamentosas utilizou-se verificadores disponíveis gratuitamente nas plataformas on-line Drug Interactions Checker Drug Information e DrugBank. A partir da análise feita nas plataformas, as interações foram classificadas segundo a intensidade dos efeitos em: "grave”, quando seus efeitos podem oferecer risco de óbito e requerem intervenção médica imediata; “moderadas”, quando seus efeitos podem ocasionar uma piora da condição clínica do paciente, tornando necessária alteração no plano medicamentoso; "leves”, classificadas como efeitos clínicos pequenos que geralmente não requerem alteração da terapia farmacológica; e "não interage”, quando os fármacos não apresentam interações entre si. ${ }^{9}$ Para aquelas combinações farmacológicas não descritas nos verificadores on-line realizou-se busca em artigos científicos, 
Análise das interações medicamentosas e perfil epidemiológico de indivíduos com... $\mid 6$

os quais não permitiram caracterizar a gravidade da interação, apenas se ela ocorria ou não (o que foi definido pelas autoras como "interage").

A pesquisa foi desenvolvida conforme as diretrizes da Resolução 466/12 do Conselho Nacional de Saúde para pesquisas realizadas em seres humanos, submetida ao Comitê de Ética em Pesquisa (CEP) da UDESC, parecer no 795133173.0000 .0118 e aprovada na data de 27 de fevereiro de 2018. A todos os participantes foi lhes guardado a confidencialidade dos dados utilizados. Na etapa de tabulação e análise dos dados o anonimato dos participantes foi garantido por meio da identificação numérica.

\section{Resultados}

A distribuição da frequência dos dados sociodemográficos, hábitos de vida e condições de saúde dos indivíduos com DM, na área de abrangência estudada, estão dispostos nas Tabelas $1 \mathrm{e}$ 2. Nos indivíduos entrevistados a média de idade foi de 68,36 anos. A informação de raça foi autodeclarada e constituída majoritariamente por brancos. Além disso, os mesmos, em sua maioria apresentaram ensino fundamental incompleto, com amostra constituída principalmente por viúvos e aposentados (Tabela 1). 
Tabela 1- Distribuição das variáveis sociodemográficas dos indivíduos com DM entrevistados. Município de Chapecó/SC, 2018.

\begin{tabular}{|c|c|c|}
\hline Variável & $\mathbf{N}$ & $\%$ \\
\hline \multicolumn{3}{|l|}{ Sexo } \\
\hline Feminino & 26 & $61,90 \%$ \\
\hline Masculino & 16 & $38,10 \%$ \\
\hline \multicolumn{3}{|l|}{ Raça } \\
\hline Brancos & 33 & $78,57 \%$ \\
\hline Negros & 8 & $19,05 \%$ \\
\hline Pardos & 1 & $2,38 \%$ \\
\hline \multicolumn{3}{|l|}{ Escolaridade } \\
\hline Ensino fundamental incompleto & 21 & $50 \%$ \\
\hline Ensino fundamental completo & 10 & $23,82 \%$ \\
\hline Ensino médio incompleto & 3 & $7,14 \%$ \\
\hline Ensino médio completo & 3 & $7,14 \%$ \\
\hline Ensino superior completo & 3 & $7,14 \%$ \\
\hline Analfabetos & 2 & $4,76 \%$ \\
\hline \multicolumn{3}{|l|}{ Estado civil } \\
\hline Viúvos & 16 & $38,10 \%$ \\
\hline Divorciados & 14 & $33,33 \%$ \\
\hline Casados & 11 & $26,19 \%$ \\
\hline Solteiros & 1 & $2,38 \%$ \\
\hline \multicolumn{3}{|l|}{ Profissão } \\
\hline Aposentado & 23 & $54,77 \%$ \\
\hline Do lar & 15 & $35,71 \%$ \\
\hline Cozinheiro (a) & 02 & $4,76 \%$ \\
\hline Motorista & 02 & $4,76 \%$ \\
\hline
\end{tabular}

Fonte: Banco de dados dos autores (2018).

O questionário contava também com perguntas sobre a ocorrência de outros agravos como Hipertensão Arterial Sistêmica (HAS), problemas visuais e obesidade, no qual o entrevistado indicava de maneira autorreferida, se condiziam com sua situação ou não. Em muitos casos o paciente possuía mais de uma comorbidade o que resultou num " $\mathrm{N}$ " total maior do que o da amostra (Tabela 2).

Outros itens do questionário, apresentados na Tabela 2, relacionam frequência das atividades físicas e cuidado com a alimentação, tabagismo e uso de bebidas alcoólicas pelos pacientes com DM. Ainda constam informações sobre os cuidados relacionados à doença, como 
Análise das interações medicamentosas e perfil epidemiológico de indivíduos com... $\mid 8$

frequência das consultas, tempo de tratamento da DM e utilização de plantas medicinais como terapia complementar.

Tabela 2 - Distribuição das variáveis relacionadas aos hábitos de vida e condições de saúde dos indivíduos com DM entrevistados. Município de Chapecó/SC, 2018.

\begin{tabular}{|c|c|c|}
\hline Variável & $\mathbf{N}$ & $\%$ \\
\hline \multicolumn{3}{|l|}{ Doenças associadas } \\
\hline Hipertensão arterial sistêmica & 34 & $80,95 \%$ \\
\hline Problemas visuais & 17 & $40,48 \%$ \\
\hline Obesidade & 09 & $21,43 \%$ \\
\hline \multicolumn{3}{|l|}{ Utilização de plantas medicinais } \\
\hline $\operatorname{Sim}$ & 25 & $59,52 \%$ \\
\hline Chá de camomila (Chamomilla recutita) & 04 & $16,00 \%$ \\
\hline Pata-de-vaca (Bauhinia forficata) & 04 & $16,00 \%$ \\
\hline Chá de marcela (Achyrocline satureioides) & 03 & $12,00 \%$ \\
\hline Amora branca (Morus alba) - relevante para o DM & 02 & $8,00 \%$ \\
\hline Chá de insulina (Cissus sicyoides) - relevante para o DM & 02 & $8,00 \%$ \\
\hline Jambolão (Syzygium cumini) - relevante para o DM & 01 & $4,00 \%$ \\
\hline Espinheira santa (Maytenus ilicifolia) - relevante para o DM & 01 & $4,00 \%$ \\
\hline Não especificaram o tipo de planta & 08 & $32,00 \%$ \\
\hline \multicolumn{3}{|l|}{ Frequência de atividade física } \\
\hline Nunca fazem exercício físico & 18 & $42,86 \%$ \\
\hline Às vezes & 13 & $30,95 \%$ \\
\hline Duas vezes por semana & 07 & $16,67 \%$ \\
\hline Três vezes por semana & 04 & $9,52 \%$ \\
\hline \multicolumn{3}{|l|}{ Controle da alimentação } \\
\hline Procura controlar & 22 & $52,38 \%$ \\
\hline Não controla & 12 & $28,57 \%$ \\
\hline Às vezes controlam & 08 & $19,05 \%$ \\
\hline \multicolumn{3}{|l|}{ Monitoramento das taxas de glicemia } \\
\hline Às vezes & 26 & $61,90 \%$ \\
\hline Diariamente & 06 & $14,29 \%$ \\
\hline Uma vez por semana & 06 & $14,29 \%$ \\
\hline A cada quinze dias & 01 & $2,38 \%$ \\
\hline Uma vez por mês & 01 & $2,38 \%$ \\
\hline Só em consultas & 01 & $2,38 \%$ \\
\hline Nunca & 01 & $2,38 \%$ \\
\hline \multicolumn{3}{|l|}{ Frequência de consultas } \\
\hline Frequentemente & 10 & $23,81 \%$ \\
\hline Quando necessário & 10 & $23,81 \%$ \\
\hline Entre $2-4$ meses & 10 & $23,81 \%$ \\
\hline A cada 6 meses & 08 & $19,05 \%$ \\
\hline
\end{tabular}




\begin{tabular}{lcc} 
Uma vez no ano & 03 & $7,14 \%$ \\
Não souberam responder & 01 & $2,38 \%$ \\
Tempo de tratamento & & \\
Menos de 5 anos & 09 & $21,43 \%$ \\
$5-10$ anos & 12 & $28,57 \%$ \\
$11-20$ anos & 11 & $26,19 \%$ \\
+ de 20 anos & 08 & $19,05 \%$ \\
Não lembram & 02 & $4,76 \%$ \\
Fumantes & & \\
Não & 41 & $97,62 \%$ \\
Sim & 01 & $2,38 \%$ \\
Consumo de bebida alcóolica & & \\
Não consomem & 35 & $83,33 \%$ \\
Consomem & 7 & $16,67 \%$ \\
\hline
\end{tabular}

Fonte: Banco de dados dos autores (2018).

Outro fator abordado foi a percepção sobre a dificuldade de cicatrização. Dos entrevistados, $7,14 \%$ sentem os efeitos da falta de cicatrização efetiva. Com relação à necessidade de realização de procedimentos em decorrência de complicações do DM foram citados a cirurgia de catarata (1 paciente), a amputação de membros (dois pacientes), e um transplante hepático. Lesões e feridas ininterruptas nos membros inferiores e superiores, foram referidos por dois dos entrevistados.

Por meio da análise dos questionários e prontuários obteve-se a relação de medicamentos usados pelos 42 pacientes. A frequência de combinações farmacológicas (fármaco 1 + fármaco 2) observada foi de 1355, as quais foram analisadas nas plataformas on-line para se determinar se havia ou não interações entre os medicamentos. Para identificar a média por paciente de combinações farmacológicas, dividiu-se o total das mesmas (1355) pela quantidade de participantes na pesquisa $(n=42)$, produziu-se assim uma média de mais de 32,26 combinações de fármacos/por paciente. Destas, as mais frequentes foram: $1^{\circ}$ ) metformina e hidroclorotiazida; $2^{\circ}$ ) metformina e sinvastatina e metformina e paracetamol; $3^{\circ}$ ) ácido acetilsalicílico e metformina, ácido acetilsalicílico e sinvastatina e paracetamol e metformina. 
Análise das interações medicamentosas e perfil epidemiológico de indivíduos com... $\mid 10$

Após análise das combinações farmacológicas, constatou-se que das 1355 combinações, $479(35,35 \%)$ resultaram em algum grau de interação medicamentosa, gerando uma média de 11,4 interações/por paciente. Entre as mais frequentes estão, hidroclorotiazida e metformina, metformina e sinvastatina, ácido acetilsalicílico e sinvastatina, ácido acetilsalicílico e metformina e paracetamol e metformina (Figura 1). Além disso, a maioria dos pesquisados relataram que retiravam os medicamentos no CSF de referência. Os demais reportaram que compravam em farmácias aqueles medicamentos de laboratórios diferentes dos fornecidos pelo SUS.

Figura 1 - As cinco interações medicamentosas mais frequentes observadas nos pacientes com DM pesquisados. Município de Chapecó/SC, 2018.

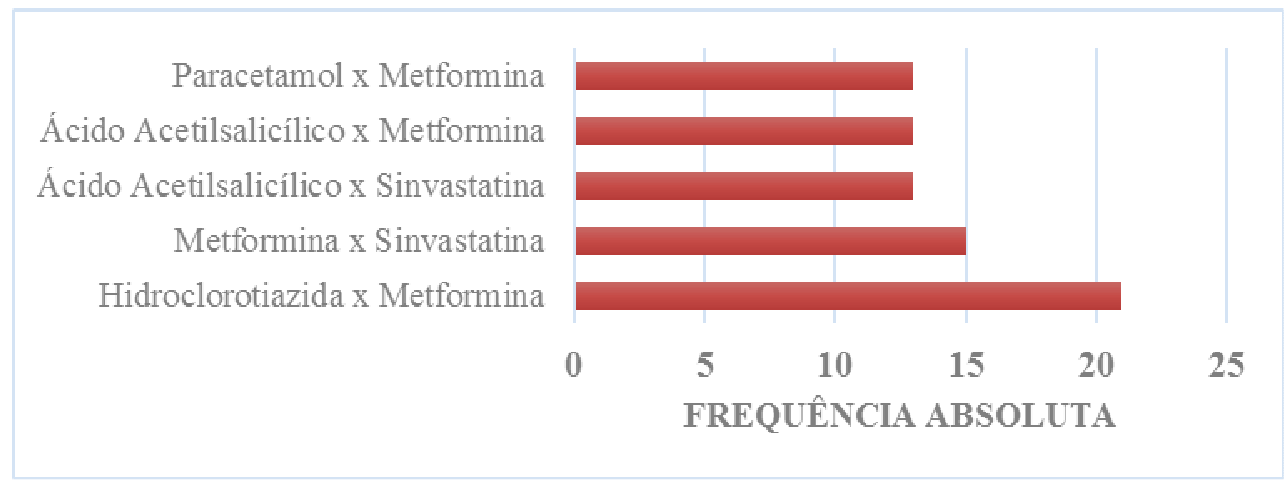

Fonte: Banco de dados dos autores (2018).

Ao analisar a ocorrência e intensidade das interações, obteve-se um percentual de 64,65\% como não interagente e totalizou-se $4,06 \%$ para as interações leves. Na categoria moderada foram $26,05 \%$ e como grave obteve-se $1,70 \%$ dos resultados. No item interage, foi contabilizado $3,54 \%$ do total (Figura 2). 
11 | Zanatta L, Cort FND, Mathias NS, Argenta C

Figura 2 - Frequência das interações analisadas segundo seu tipo. Município de Chapecó/SC, 2018.

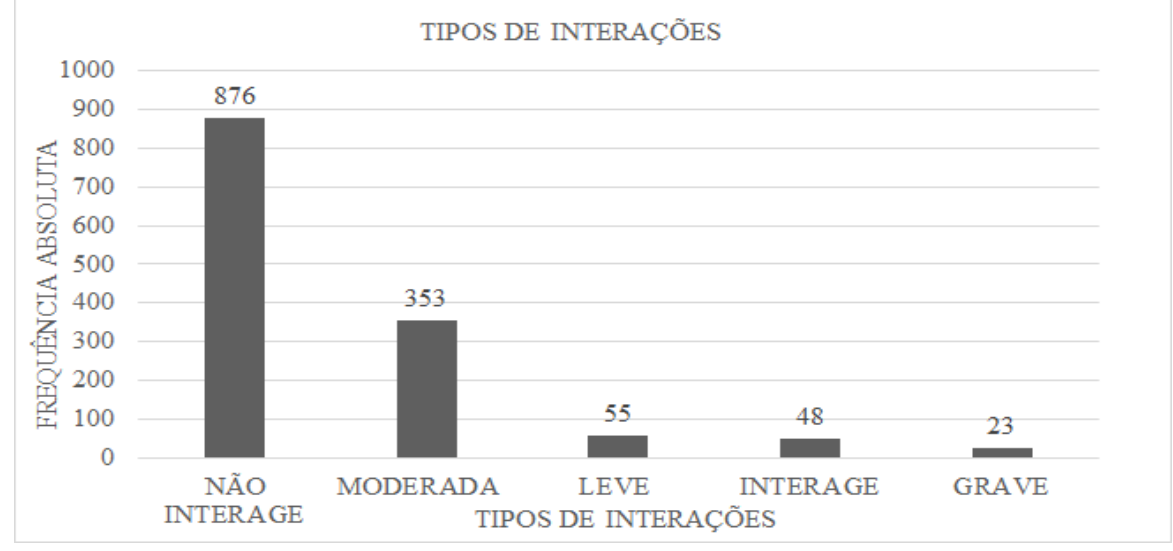

Fonte: Banco de dados dos autores (2018).

Nas interações graves, as combinações que mais predominaram foram entre sinvastatina e anlodipino, atenolol e clonidina e losartana e espironolactona (Figura 3A). Dentre as interações medicamentosas do tipo moderada, a combinação de hidroclorotiazida e metformina foi a mais observada (Figura 3B). Quanto às interações leves, as mais frequentes foram furosemida e ácido acetilsalicílico e ácido acetilsalicílico e atenolol (Figura 3C). 
Análise das interações medicamentosas e perfil epidemiológico de indivíduos com... $\mid 12$

Figura 3 - Interações mais frequentemente observadas de acordo com as suas gravidades (a) graves, b) moderadas e c) leves. Município de Chapecó/SC, 2018.

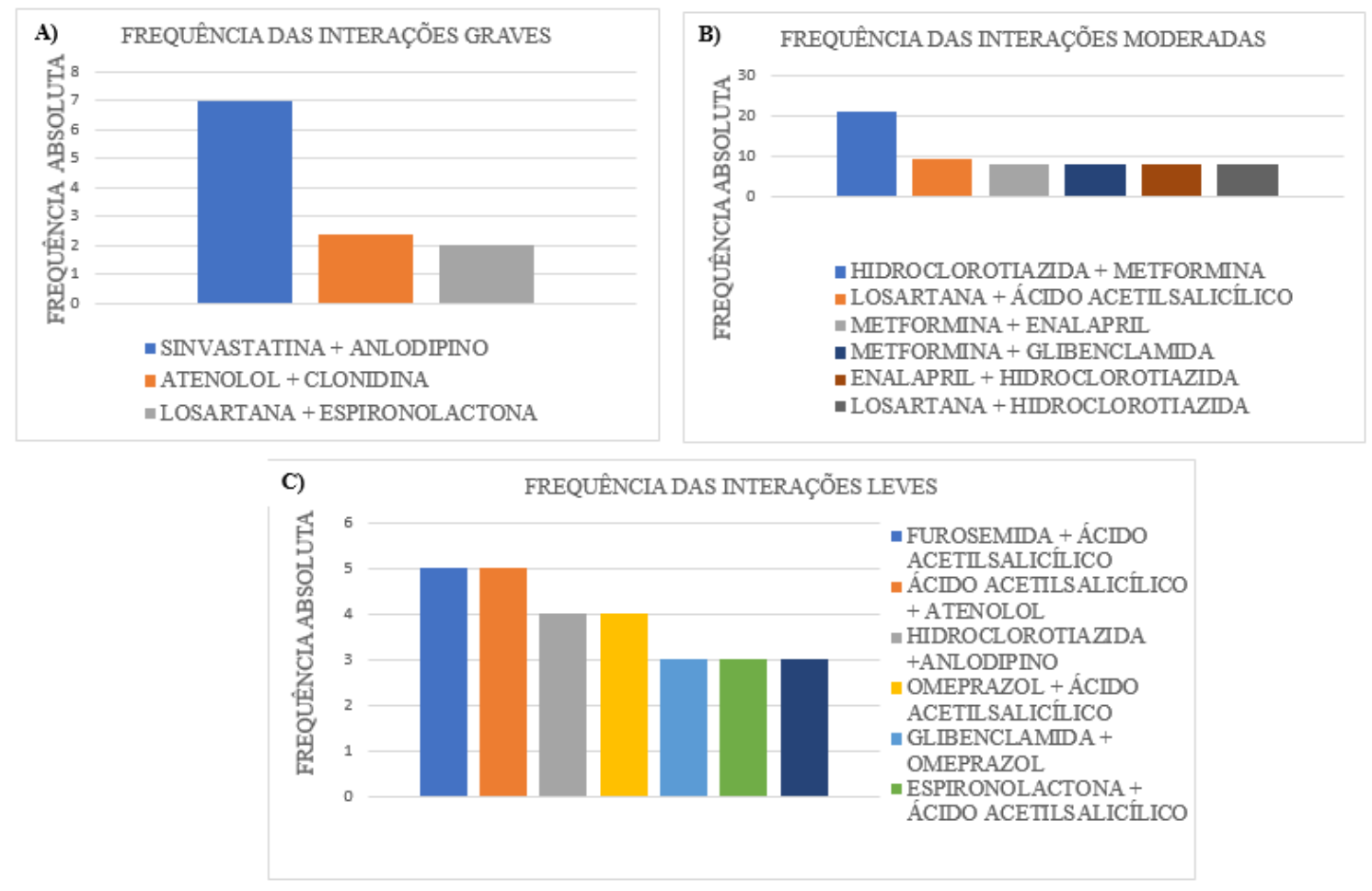

Fonte: Banco de dados dos autores (2018).

A contagem das associações de medicamentos classificadas apenas como interagentes resultou em três aparições para metformina e nimesulida, bem como, hidroclorotiazida e nimesulida, nimesulida e atenolol, hidroclorotiazida e Dorflex ${ }^{\circledR}$ (dipirona, cafeína e citrato de orfenadrina), e hidroclorotiazida e dipirona. Das combinações de fármacos que não interagem, a de maior frequência se deu entre metformina e sinvastatina com quinze vezes. Com treze vezes, aparecem sinvastatina e ácido acetilsalicílico, ácido acetilsalicílico e metformina, metformina e paracetamol.

\section{Discussão}

Fisiologicamente, homens e mulheres apresentam inúmeras diferenças, bem como seus hábitos e costumes de vida podem diferenciar, tornando-os mais ou menos susceptíveis a determinadas doenças. ${ }^{10}$ Diferente do observado neste estudo, a nível mundial os homens são os 
mais acometidos pelo DM. ${ }^{10}$ A idade é mais um dos fatores que contribui para o desenvolvimento do DM. Neste estudo, a maioria eram idosos diagnosticados com DM tipo 2 corroborando com dados nacionais que sugerem uma prevalência de pacientes com DM de aproximadamente $20 \%$ na faixa etária de 35 a 79 anos, embora, a Federação Internacional de Diabetes (IDF) estime $8,8 \%$ na população mundial. ${ }^{1}$

No DM tipo 2, o fator raça é também considerado uma condição de risco para o seu desenvolvimento, sendo os negros, hispânicos ou índios Pima, os mais suscetíveis a este agravo. ${ }^{1}$ Além disso, o tipo de raça do paciente pode estar relacionado a diferentes condições como o acesso e o uso dos serviços de saúde, além de estar vinculado a fatores econômicos, de hábitos e comportamentos. ${ }^{11}$ Da mesma forma, o nível de escolaridade é fator de risco para a doença, pois influencia a disponibilidade de acesso às informações. ${ }^{12}$ Neste contexto, este estudo demonstra que $50 \%$ dos pacientes têm ensino fundamental incompleto, sugerindo isto como fator causador das falhas no autocuidado, como baixa frequência nas consultas e monitorização da glicemia e a falta de prática de exercícios físicos.

O tabagismo é também considerado uma ameaça para o desenvolvimento de DM, já que estudos relacionam esta combinação a fatores como: altos níveis de cortisol, elevados marcadores de inflamação e de estresse oxidativo e obesidade centralizada. Além disso, a nicotina restringe claramente a secreção da insulina. ${ }^{1}$ A mesma cautela se aplica ao consumo de bebidas alcoólicas já que o álcool afeta tanto a alimentação quanto a glicemia. ${ }^{1}$

As doenças associadas ao DM, mais reportadas na pesquisa foram a HAS, retinopatia e obesidade. A HAS é uma comorbidade comum do DM. O indivíduo que apresenta ambas possui diversos mecanismos fisiopatológicos que os deixa com um maior risco de desenvolver agravos cardiovasculares e renais. ${ }^{1}$ A retinopatia diabética é uma das mais relevantes condições que causam perda visual irreversível a nível mundial ${ }^{1}$ e a obesidade está associada à uma elevação da 
Análise das interações medicamentosas e perfil epidemiológico de indivíduos com... $\mid 14$

demanda de insulina devido à resistência dos tecidos, o que ocasiona aumento na glicemia e consequente hiperinsulinemia. ${ }^{13}$

O DM é um agravo crônico, sem terapêuticas desenvolvidas para a cura até o momento. Por este motivo, fatores como alto custo e efeitos adversos indesejados provocados pelos medicamentos, propiciam a busca por substâncias naturais que sejam benéficas na redução da glicemia. Porém, é importante observar que muitas plantas medicinais podem interagir com antidiabéticos e interferir na eficácia do tratamento. ${ }^{14}$ Dentre os chás usados pelo efeito hipoglicemiante pelos pesquisados e que se têm comprovação científica, estão o chá de Bauhinia variegata e Bauhinia forficata, espécies conhecidas também como pata-de-vaca, de jambolão (Eugenia jambolana ou Syzygium cumini), de folha de amoreira (Morus alba L.) e o chá de insulina (Cissus sicyoides). ${ }^{1,15-16}$ De todas as plantas citadas na pesquisa, a espinheira santa (Maytenus ilicifolia) foi a única que não apresentou evidências científicas comprovando seu efeito hipoglicêmico.

Outras práticas eficazes no controle da glicemia e recomendadas pela Sociedade Brasileira de Diabetes $^{1}$ são a realização frequente de atividade física e a adoção de uma alimentação adequada. ${ }^{1}$ Além disso, para um melhor resultado terapêutico é importante realizar a monitorização da glicemia. Porém, muitas vezes, esse controle é afetado por falta de recursos, indisponibilidade de material na rede de forma gratuita, incapacidade do paciente de ir até o CSF de referência, desgaste dos locais de aplicação entre outros fatores. ${ }^{17}$

Em relação aos dados sobre as potenciais interações medicamentosas nesse grupo de indivíduos com DM, os mesmos corroboram dados da literatura que apontam as interações de risco moderado como as mais frequentes entre pacientes com DM, e entre elas está a associação hidroclorotiazida e metformina. ${ }^{18-19}$ Além disso, percebe-se que as mais prevalentes foram aquelas cujos fármacos são utilizados para tratar as comorbidades referidas ou então decorrentes da 
automedicação, que normalmente envolve analgésicos e anti-inflamatórios. Esta última classe está entre as principais envolvidas em interações medicamentosas em pacientes com DM. ${ }^{20}$

Analisando as interações medicamentosas do tipo "graves”, mais frequentes entre os pesquisados, observou-se o predomínio da combinação de sinvastatina e anlodipino. Deve-se atentar ao aumento significativo de sinvastatina na corrente sanguínea quando ocorre essa associação. Como principais complicações estão a hepatotoxidade e rabdomiólise. ${ }^{21}$ A terapia pode ser descontinuada caso haja sintomas como urina escura, náusea, vômito, icterícia, entre outros. ${ }^{21} \mathrm{Na}$ utilização de atenolol e clonidina há probabilidade de diminuição na pressão arterial, e o paciente pode apresentar sintomas como dores de cabeça, tontura e episódios de síncope. No caso da espironolactona e losartana pode ocorrer hipercalemia, com evolução para insuficiência renal e parada cardíaca. ${ }^{211}$ Cabe destacar que as consequências dessas interações graves são potencialmente fatais ou capazes de causar danos irreversíveis ao paciente. ${ }^{9}$

Quanto às interações moderadas, hidroclorotiazida e metformina foi a combinação que prevaleceu, devendo-se observar os níveis de glicose sanguínea que podem ser alterados. $\mathrm{Na}$ associação de metformina e enalapril, pode ocorrer o aumento do efeito da metformina com consequente desenvolvimento de hipoglicemia. ${ }^{22}$

Nas interações leves, a combinação mais frequente foi entre furosemida e ácido acetilsalicílico. Nessa categoria os critérios clínicos não são extremamente relevantes em todos os casos. No caso dos salicilatos serem utilizados em dosagens anti-inflamatórias, pode ocorrer redução dos efeitos diurético e natriurético nos diuréticos de alça. Geralmente não causam danos e nem exigem que haja alterações na terapia. ${ }^{8,21}$ Ademais, as interações leves não necessitam de mudança na terapêutica, nem intervenção imediata por não serem clinicamente relevantes. ${ }^{23}$

Vale destacar que muitas das interações medicamentosas analisadas foram decorrentes da automedicação, principalmente quando envolvem analgésicos e anti-inflamatórios. A mesma está associada a fatores de aspecto financeiro, político e cultural e vem sendo aderido por boa 
Análise das interações medicamentosas e perfil epidemiológico de indivíduos com... $\mid 16$

parte da população. ${ }^{24}$ Em virtude da variedade de produtos fabricados pela indústria, a facilidade de comercialização, a gama de informações médicas obtidas de forma acessível por meio de blogs, sites e redes sociais, a automedicação vem se constituindo um problema de saúde pública, despertando nos órgãos de prevenção à saúde preocupação. Deste modo, exigindo desenvolvimento de estratégias ligadas ao não incentivo equivocado de medicamentos. ${ }^{24}$

Com base nesses achados percebe-se que o acompanhamento do paciente precisa ocorrer de maneira integral, em conjunto com a equipe para que a definição do tratamento seja conivente com seus distúrbios. Na consulta de enfermagem, optar por enfatizar as questões de hábitos de vida que interferem no processo de tratamento do paciente, estimulando a assiduidade nas consultas e o controle e monitorização adequada dos níveis glicêmicos. Além disso, orientando quanto ao uso de chás e os riscos da automedicação. ${ }^{5}$ Quando a procura pelos serviços de saúde for fora do contexto de inserção do usuário, importante orientar o paciente a carregar junto nas consultas, receituários ou até mesmo as caixas de medicamentos para não haver duplicidade nas prescrições nem o risco de interação entre medicamentos.

\section{Conclusão}

Os resultados obtidos nesse estudo permitiram estabelecer o perfil epidemiológico dos pacientes com DM atendidos em um CSF do município de Chapecó/SC. A partir desses dados, a equipe de saúde, geralmente liderada pelo enfermeiro, pode estabelecer medidas de cuidado voltadas para o controle glicêmico desses pacientes considerando sua realidade econômica e social.

A análise das combinações farmacológicas, nesse grupo de pacientes com DM, chama atenção pela intensidade de efeitos diversos que podem surgir em decorrência das interações entre os fármacos. Esses dados ao mesmo tempo que geram um "alerta" da necessidade de maior cuidado na prescrição e dispensação dos medicamentos para esse público, também permitem uma reflexão de como a enfermagem pode atuar para evitar esses danos. Sendo a automedicação 
um dos fatores resultantes no número de interações, é de relevância o cuidado, o acompanhamento intrínseco e a realização de orientações aos pacientes quanto a temática, já que a mesma influencia no curso do seu tratamento.

Perante a isso, o enfermeiro tem papel nesse processo por meio do acompanhamento contínuo, realização de orientações quanto ao uso dos fármacos, inclusão de grupos para socialização das vivências, integração com a equipe multiprofissional para atuar em todos os níveis de saúde. O controle dos fatores de risco associados ao DM, por meio de medidas de promoção da saúde, podem contribuir para diminuir a incidência da doença e de suas complicações crônicas, bem como para a redução dos custos gerados ao sistema.

Apesar das limitações metodológicas do estudo, principalmente quanto ao tamanho da amostra e acesso a sistemas gratuitos de avaliação de interações medicamentosas, os resultados aqui descritos permitirão redirecionar a prática dos profissionais que atendem essa população, nos Centros de Saúde da Família do Município, no intuito de diminuir os riscos associados ao tratamento farmacológico dos mesmos.

\section{Referências}

1. Sociedade Brasileira de Diabetes. Diretrizes da Sociedade Brasileira de Diabetes 2017-2018. São Paulo (SP): Clannad; 2017 [acesso em 2019 abr 22]. Disponível em: https://www.diabetes.org.br/profissionais/images/2017/diretrizes/diretrizes-sbd-2017-2018.pdf

2. Ribeiro Neto LM, Costa Junior VL, Crozara MA. Interações medicamentosas potenciais em pacientes ambulatoriais. Mundo da Saúde (Online). 2017;41(1):107-15. doi: http://dx.doi.org/10.15343/01047809.20174101107115

3. Santos AM, Carneiro LS, Chaud LCS, Urias GMPC, Brum HCC. Estudo do perfil farmacoterapêutico de pacientes idosos portadores de diabetes tipo II. Rev Ciênc Saúde [Internet]. 2016 dez [acesso em 2019 abr 05];1(3):24-33. Disponível em: http://revistaeletronicafunvic.org/index.php/c14ffd10/article/view/42/61

4. Secoli SR. Polifarmácia: interações e reações adversas no uso de medicamentos por idosos. Rev Bras Enferm. 2010 jan-fev;63(1):136-40. doi: http://dx.doi.org/10.1590/S0034-71672010000100023 
Análise das interações medicamentosas e perfil epidemiológico de indivíduos com... $\mid 18$

5. Oliveira IF, Sousa KMO, França EMDM, Lima CB, Barreto MA. Contribuição do enfermeiro na assistência à pessoa idosa com diabetes mellitus. Rev Temas Saúde [Internet]. 2016 [acesso em $2020 \mathrm{fev}$ 13];16(2):518-34. Disponível em: http://temasemsaude.com/wp-content/uploads/2016/08/16229.pdf

6. Hair JF Jr, Black WC, Babin BJ, Anderson RE, Tathan RL. Análise multivariada de dados. 6ª ed. Porto Alegre: Bookman; 2009.

7. Dresch AP, Amador TA, Heineck I. Conhecimento dos pacientes sobre medicamentos prescritos por odontólogos no sul do Brasil. Ciênc Saúde Colet. 2016;21(2):475-84. doi: https://doi.org/10.1590/141381232015212.17732014

8. Lima ML. Avaliação da prevalência da automedicação no município de Nova Olinda- CE [monografia]. Nova Olinda: Escola de Saúde Pública do Ceará; 2007 [acesso em 2020 fev 13]. Disponível em: https://studylibpt.com/doc/1464255/avalia\%C3\%A7\%C3\%A3o-da-preval\%C3\%AAncia-daautomedica\%C3\%A7\%C3\%A3o-no-munic\%C3\%ADpio-de...

9. Lima TAM, Furini AAC, Atique TSC, Done PD, Machado RLD, Godoy MF. Análise de potenciais interações medicamentosas e a reações adversas a anti-inflamatórios não esteroidais em idosos. Rev Bras Geriatr Gerontol. 2016 Jun;19(3):533-44. doi: http://dx.doi.org/10.1590/1809-98232016019.150062

10. Hospital Sírio Libanês. Diabetes é mais comum nas mulheres do que em homens [Internet]. São Paulo; 2017 [acesso em 2017 abr 23]. Disponível em: https://hospitalsiriolibanes.org.br/suasaude/Paginas/diabetes-mais-comum-nas-mulheres-que-nos-homens.aspx

11. Moretto MC, Fontaine AM, Garcia CAMS, Neri AL, Guariento ME. Associação entre cor/raça, obesidade e diabetes em idosos da comunidade: dados do Estudo FIBRA. Cad Saúde Pública. 2016;32(10):e0081315. doi: http://dx.doi.org/10.1590/0102-311X00081315

12. Costa LJSL, Machado ALG, Silva AZ, Braga CR, Costa JN, Formiga LMF. Self-care of young adults with type 2 diabetes mellitus. Rev Enferm UFPE On Line [Internet]. 2016 Nov [cited 2019 Oct 09];10(11):3875-82. Available from:

https://periodicos.ufpe.br/revistas/revistaenfermagem/article/view/11468

13. Flor LS, Campos MR. Prevalência de diabetes mellitus e fatores associados na população adulta brasileira: evidências de um inquérito de base populacional. Rev Bras Epidemiol. 2017 Mar;20(1):16-29. doi: http://dx.doi.org/10.1590/1980-5497201700010002

14. May M, Schindler C. Clinically and pharmacologically relevant interactions of antidiabetic drugs. Ther Adv Endocrinol Metab. 2016;7(2):69-83. doi: https://doi.org/10.1177/2042018816638050

15. Alvarenga CF, Lima KMN, Mollica LR, Azevedo LO, Carvalho C. Uso de plantas medicinais para o tratamento do diabetes mellitus no vale do Paraíba-SP. Rev Ciênc Saúde [Internet]. 2017 [acesso em 2019 abr 22];2(2):36-44. Disponível em: http://revistaeletronicafunvic.org/index.php/c14ffd10/article/view/84/73 
16. Xavier AT, Nunes JS. Tratamento de diabetes mellitus com plantas medicinais. Rev Cient FAEMA. 2018 jun;9(N Esp):603-9. doi: https://doi.org/10.31072/rcf.v9iedesp.616

17. Tian S, Tang M, Zhao B. Current anti-diabetes mechanisms and clinical trials using Morus alba L. J Tradit Chin Med. 2016 Jan;3(1):3-8. doi: https://doi.org/10.1016/j.jtcms.2016.04.001

18. Lima RF, Machado AV, Rabelo FM, Naves JOS, Lavich TR, Dulliu J. Interações medicamentosas potenciais em diabéticos tipo 2 participantes de um programa de educação em saúde. Infarma [Internet]. 2015 jul [acesso em 2020 fev 16];27(3):160-7. Disponível em: http://revistas.cff.org.br/?journal=infarma\&page=article\&op=view\&path\%5B\%5D=747\&path\%5B\%5D=pdf

19. Prado MAMB, Francisco PMSB, Barros MBA. Diabetes em idosos: uso de medicamentos e risco de interação medicamentosos. Ciênc Saúde Colet. 2016;21(11):3447-58. doi: https://doi.org/10.1590/1413812320152111.24462015

20. Ikäheimo I, Karjalainen M, Tiihonen M, Haanpää M, Kautiainen H, Saltevo J, et al. Clinically relevant drug-drug interactions and the risk for drug adverse effects among home-dwelling older persons with and without type 2 diabetes. J Clin Pharm Ther. 2019;44(5):735-41. doi: https://doi.org/10.1111/jcpt.12854

21. Costa MA, Tasca RS, Apolinário APA, Borghi L. Caracterização do uso de sinvastatina pelos pacientes da terceira idade. Rev Cient Ágape [Internet]. 2018 [acesso em 2019 abr 22];1(1):1-13. Disponível em: http://www.revistaagape.com.br/index.php/revistaagape/article/view/4/6

22. Rempel C, Goettert MI, Strohschoen AAG, Carreno I, Manfroi M, Moreschi C. Análise da medicação utilizada por diabéticos e hipertensos. Cad Pedagóg [Internet]. 2015 [acesso em 2020 maio 11];12(1):24152. Disponível em: http://www.univates.br/revistas/index.php/cadped/article/viewFile/948/936

23. Cruz HL, Mota FKC, Araújo LU, Bodevan EC, Seixas SRS, Santos DF. The utility of the records medical: factors associated with the medication errors in chronic disease. Rev Latinoam Enferm. 2017;(25):e2967:1-10. doi: http://dx.doi.org/10.1590/1518-8345.2406.2967

24. Arrais PSD, Fernandes MEP, Pizzol TSD, Ramos LR, Mengue SS, Luiza VL, et al. Prevalência da automedicação no Brasil e fatores associados. Rev Saúde Pública. 2016;50(Suppl 2):13s. doi: http://dx.doi.org/10.1590/s1518-8787.2016050006117

\section{Autor correspondente}

Leila Zanatta

E-mail: leila.zanatta@udesc.br

Endreço: Rua Sete de Setembro, 91-D - Centro, Chapecó-SC.

CEP: 89801-140 
Análise das interações medicamentosas e perfil epidemiológico de indivíduos com... | 20

\section{Contribuições de Autoria}

\section{1 - Leila Zanatta}

Contribuições: concepções e planejamento do projeto de pesquisa, análise dos dados, redação e revisão crítica.

\section{2 - Fernanda Norbak Dalla Cort}

Contribuições: concepções e planejamento do projeto de pesquisa, obtenção e análise dos dados e redação.

\section{3- Nathália Silva Mathias}

Contribuições: concepções e planejamento do projeto de pesquisa, obtenção e análise dos dados e redação.

\section{4 - Carla Argenta}

Contribuições: redação e revisão crítica.

\section{Como citar este artigo}

Zanatta L, Cort FND, Mathias NS, Argenta C. Análise das interações medicamentosas e perfil epidemiológico de indivíduos com diabetes mellitus na atenção primária. Rev. Enferm. UFSM. 2020 [Acesso em: Anos Mês Dia]; vol.10 e47: 1-20. DOI:https://doi.org/10.5902/2179769240175 